\title{
Pervasive non-CpG methylation at zebrafish mosaic satellite repeats
}

\author{
Samuel E Ross ${ }^{1,2}$, Allegra Angeloni ${ }^{1,2}$, Alex de Mendoza ${ }^{3}$, Ozren Bogdanovic ${ }^{1,4}$ \\ 1 Genomics and Epigenetics Division, Garvan Institute of Medical Research, Sydney, New South Wales, \\ 2010, Australia \\ 2 St Vincent's Clinical School, Faculty of Medicine, University of New South Wales, Sydney, New South \\ Wales, 2010, Australia. \\ 3 School of Biological and Chemical Sciences, Queen Mary University of London, London, E1 4NS, \\ United Kingdom. \\ 4 School of Biotechnology and Biomolecular Sciences, University of New South Wales, Sydney, New \\ South Wales, 2052, Australia. \\ Correspondence to: o.bogdanovic@garvan.org.au
}




\begin{abstract}
In vertebrates, DNA methylation predominantly occurs at CG dinucleotides even though widespread non-CG methylation $(\mathrm{mCH})$ has been reported in mammalian embryonic and neural cells. Unlike in mammals, where $\mathrm{mCH}$ is found enriched at $\mathrm{CAC} / \mathrm{G}$ trinucleotides and is tissue-restricted, we find that zebrafish embryos as well as adult somatic and germline tissues display robust methylation enrichment at TGCT positions associated with mosaic satellite repeats. These repeats reside in $\mathrm{H} 3 \mathrm{~K} 9$ me3-marked heterochromatin and display $\mathrm{mCH}$ reprogramming coincident with zygotic genome activation. Altogether, this work provides insight into a novel form of vertebrate $\mathrm{mCH}$ and highlights the substrate diversity of vertebrate DNA methyltransferases.
\end{abstract}

Keywords: DNA methylation, embryogenesis, zebrafish, repetitive elements 


\section{Background}

Methylation of cytosines within the CG dinucleotide context is the most abundant DNA modification in vertebrate genomes [1]. CG methylation (mCG) is found in all vertebrate cell types and is known to participate in long-term gene silencing processes [2]. Nevertheless, methylation of other cytosine dinucleotides $(\mathrm{mCH}, \mathrm{H}=\mathrm{T}, \mathrm{G}, \mathrm{A})$ has also been described. $\mathrm{mCH}$ (also referred to as non-CG methylation), is most commonly found in mammalian embryonic stem cells (ESCs) and in the brain, however, $\mathrm{mCH}$ has been identified at low levels in almost all human tissues [3-5]. The functions of $\mathrm{mCH}$ in gene regulation remain unresolved to date. In mammalian brains, $\mathrm{mCH}$ levels are inversely correlated with transcription of the associated gene, whereas this pattern appears to be the opposite in ESCs [4]. $\mathrm{mCH}$ within the CA context has also been previously observed at major satellite repeats in mouse ESCs but not in differentiated cells [6]. In mammals, $\mathrm{mCH}$ accumulates during nervous system development, and displays considerable remodelling during iPSC reprogramming and direct conversion of fibroblasts to neurons [4,7-9]. Unlike $\mathrm{mCG}, \mathrm{mCH}$ methylation is not maintained after cell division by DNMT1, and therefore requires constant activity of de novo DNMT3 enzymes. $\mathrm{mCH}$ deposition is carried out by both DNMT3A and DNMT3B, mostly at CAC or CAG trinucleotides respectively, suggestive of significant sequence specificity during DNMT3 recruitment [10]. Preferential methylation at CAC trinucleotides has also been recently reported as a conserved feature across vertebrates, from lamprey to mammalian brains [11]. However, to date, other forms of $\mathrm{mCH}$ have not been identified in vertebrates.

\section{Results and discussion}

To evaluate the presence of non-CG methylation during zebrafish development we reanalyzed whole genome bisulfite sequencing (WGBS) data to obtain genotypecorrected $\mathrm{mCH}$ profiles of $80 \%$ epiboly (gastrula), 24 hours post fertilization (hpf, pharyngula), 48hpf (hatching) embryos, and adult brain tissue (bisulfite conversion $>99,5 \%$ ) [12]. All samples showed only a minor elevation of methylation at CA dinucleotides compared to the unmethylated lambda genome spike in control, except for the brain sample which had a moderate 1.5 and 2 fold increase at both CT and CA dinucleotides, respectively (Fig. 1a). This is in line with the reported mCA enrichments in the zebrafish forebrain [11]. However, when motif calling was performed on the most highly methylated sites in the $\mathrm{CH}$ context, the top enriched sequence was consistently CATGCTAA, with methylation occurring at the TGCT tetranucleotide (Fig. $\mathbf{1 b}$ and Additional File 1:Figure S1). Methylation was almost exclusively detected on the strand displaying the 5'-TGCT-'3' motif (>75\%) rather than on its reverse complement (5'-AGCA-3'), suggestive of considerable strand specificity during DNMT targeting as well as lack of symmetry typical of CG methylation (Additional File 1:Figure S1). Many of these nucleotides displayed substantial $\mathrm{mCH}$ of $>10 \%$, particularly at repetitive 
elements where this motif was found to contain the highest levels of $\mathrm{mCH}$ and had a notable increase in methylation at later stages of development and in the brain (Fig. 1c and Additional File 1:Figure S1). When the repetitive sites of the genome bearing the CATGCTAA motif were annotated, we found that more than $65 \%$ of these sites are located in MOSAT_DR mosaic satellite repeats (GenBank ID: DP000237.1, Fig. 1d,e). Hereafter we refer to this motif as the MOSAT motif. Due to potential mappability issues of repetitive elements, we re-mapped uniquely mapping reads covering MOSAT_DR repeats, tolerating 0 mismatches across the entire read (Additional File 1:Figure S2 and Additional File 2). Even under such stringent mapping conditions we observe substantial coverage of MOSAT_DR repeats and confirm the strand bias associated with TGCT methylation (Additional File 1:Figure S2). Additionally, to confirm that this form of methylation is not the result of a sequence-specific bias of bisulfite conversion [13], we generated enzymatic methylation sequencing (EM-seq) base-resolution libraries of two biological replicates of 24hpf embryos [14 ](Additional File 1:Table S1). The top methylated MOSAT motifs identified in either EM-seq or WGBS showed proportionate methylation levels when compared to the orthogonal approach (Fig. 1f,g and Additional File 1:Figure S3). We therefore conclude that $\mathrm{mCH}$ associated with MOSAT motifs is not due to low bisulfite conversion efficiencies or mappability issues associated with these repetitive regions; MOSAT_DR is an actively $\mathrm{CH}$-methylated satellite repeat in zebrafish.

MOSAT_DR elements containing the MOSAT motif are found almost exclusively at CGdepleted introns and intergenic regions (Fig. 2a). The scarcity of CG dinucleotides in these elements suggests that this type of methylation is unlikely to just be a byproduct of DNMT activity targeting CG sites. In both embryos and adult tissues there is an overall positive correlation between the number of MOSAT motifs and mean $\mathrm{mCH}$ levels of the gene observed at all stages, suggesting a major contribution of TGCT methylation to $\mathrm{mCH}$ gene body patterning (Fig. 2b, Additional File 1: Figure S4). Gene ontology (GO) analysis [15] of MOSAT motif-containing genes demonstrated a significant enrichment for terms associated with neuronal function and in particular synaptic function, in agreement with previous reports on neural $\mathrm{mCH}$ enrichment in mammals [4] (Additional File 1: Figure S4). Given the observation that long genes can be enriched and sensitive to $\mathrm{mCH}$ levels in mice and human brains [16,17], we investigated the length of MOSAT motif-containing genes and found them to be, on average, significantly longer than all genes as well as neural genes (Additional File 1: Figure S4). Moreover, MOSAT motif-containing genes do not appear to be constitutively expressed. They exhibit expression in a diverse range of tissues many of which are neural by origin, however, their expression is not exclusively limited to the nervous system [18] (Additional File 1: Figure S4). 
Numerous reports have previously described the developmental dynamics of zebrafish CG methylation in somatic and germline tissues [12,19-27]. To further investigate the developmental dynamics of $\mathrm{mCH}$, we reanalyzed base-resolution profiles of liver, sperm, egg, 32 cell stage, 64 cell stage, sphere, germring, and shield embryos [21,24] (Additional File 1: Table S2). $\mathrm{mCH}$ levels of MOSAT motifs in commonly covered MOSAT_DR elements (8064 sites from 3091 elements) revealed high $\mathrm{mCH}$ levels in adult germ cells, cleavage stage embryos and late stage embryos/adult tissues but low levels surrounding the sphere stage, which corresponds to the major wave of zygotic genome activation-ZGA (Fig. 2 and Additional File 1:Figure S5). This is in accord with steady state transcript abundance of zebrafish de novo DNMT3 enzymes that are low in post-fertilization embryos and higher during later developmental stages, indicating their possible roles in the maintenance of this unique form of methylation [28,29] (Additional File 1:Figure S5). The observed temporal $\mathrm{mCH}$ dynamics are independent of $\mathrm{mCG}$ changes at these regions and are uncoupled from global developmental mCG patterning in zebrafish, which progressively increases from 16 cell stage to match the paternal methylome at the ZGA onset [20,21] (Additional File 1:Figure S5). However, the dynamic is similar to what has been observed during mammalian DNA methylome reprogramming, where $\mathrm{mCG}$ inherited from male and female germ cells is erased during preimplantation development and re-established during gastrulation in all embryonic layers [30-33]. It is also resemblant of overall mammalian $\mathrm{mCH}$ dynamics, which is characterized by gradual dilution of oocyte-inherited $\mathrm{mCH}$ during cleavage stages and its gradual re-establishment in the nervous system. [4,31,34].

Finally, we wanted to assess the regulatory environment of $\mathrm{mCH}$-methylated MOSAT motifs. To that end, we analyzed ChIP-seq data of H3K9me3, H3K27me3, H3K36me3, H3K27ac, H3K4me1, and H3K4me3 histone modifications [35-37]. We found a clear enrichment of H3K9me3 at methylated MOSAT_DR elements, whereas no other histone modification displayed any signal at these sites (Fig.2d-e and Additional File1:Figure S5). H3K9me3 has been shown to mark the zebrafish genome pre-ZGA and to progressively increase from ZGA onwards [35,38], however, we find that at a sub-population of MOSAT_DR sites, H3K9me3 is stable during development (256 cell to shield) (Additional File1:Figure S5). This contrasts the observation in mammals, where $\mathrm{H} 3 \mathrm{~K} 9 \mathrm{me} 3$ and $\mathrm{mCH}$ are generally inversely correlated at CG-poor regions $[8,29,39]$. To interrogate if $\mathrm{H} 3 \mathrm{~K} 9 \mathrm{me} 3$ could play a role in recruiting or maintaining $\mathrm{mCH}$ at MOSAT repeats, we investigated the correlation between $\mathrm{mCH}$ and $\mathrm{H} 3 \mathrm{~K} 9 \mathrm{me} 3$ through development. We observe a strong positive correlation at the 32 cell stage, $80 \%$ epiboly and adult brain $(r>0.6)$ where $\mathrm{mCH}$ is enriched, and a lower correlation at stages surrounding the ZGA $(r>0.35)$, when $\mathrm{mCH}$ is being reprogrammed (Fig. $2 f$ and Additional File1:Figure S5). A possible explanation for this correlation could be that $\mathrm{mCH}$ is re-established by means of $\mathrm{H} 3 \mathrm{~K} 9 \mathrm{me} 3$ driven recruitment at these regions. This 
could be facilitated by DNMT3 enzymes as H3K9me3 can recruit DNMT3 to satellite repeat regions in ESCs [40]. Also, a previous report demonstrated H3K9me3 and DNMT3 cooperation during zebrafish embryogenesis [41]. These findings thus merit further investigation into the possible relationships between heterochromatin and $\mathrm{mCH}$, suggesting that the inverse correlation between $\mathrm{mCH}$ and $\mathrm{H} 3 \mathrm{~K} 9 \mathrm{me} 3$ observed in mammals might not be conserved, or that zebrafish display a lineage-specific innovation.

\section{Conclusions}

Our meta-analysis of $\mathrm{mCH}$ in developing zebrafish revealed enrichment of TGCT methylation within mosaic satellite repeats. These motifs were highly methylated in adult germ cells, pre-ZGA embryos, post gastrulation embryos, and adult brain and liver. However, methylation was diminished between the sphere stage and late gastrulation, suggestive of developmental remodeling coincident with ZGA onset thus displaying similarities to mammalian methylome reprogramming. We also found $\mathrm{mCH}$ at those sites to be highly correlated with $\mathrm{H} 3 \mathrm{~K} 9$ me3 enrichment during early development in contrast to what has been observed in mammals. In conclusion, we have described a novel type of vertebrate $\mathrm{mCH}$, one with a unique sequence specificity, which undergoes developmental loss and re-establishment at H3K9me3-marked heterochromatin regions.

\section{Methods}

\section{Zebrafish usage and ethics}

Zebrafish work was approved by the Garvan Institute of Medical Research Animal Ethics Committees under AEC approval 17/22. All procedures performed complied with the Australian Code of Practice for Care and Use of Animals for Scientific Purposes. Adult wild type (AB/Tuebingen) Danio rerio (zebrafish) were bred in a 1 male:1 female ratio. Embryos were collected 0 hours post-fertilization (hpf) and incubated in 1X E3 medium $(0.03 \% \mathrm{NaCl}, 0.005 \% \mathrm{CaCl} 2,0.0013 \% \mathrm{KCl}, 99.9557 \% \mathrm{H} 2 \mathrm{O}, 0.008 \%$ $\mathrm{H} 14 \mathrm{MgO} 11 \mathrm{~S})$ for 24 hours at $28.5^{\circ} \mathrm{C}$.

\section{Genomic DNA extraction}

$24 \mathrm{hpf}$ embryos were dechorionated using $1 \mathrm{mg} / \mathrm{mL}$ Pronase (Sigma-Aldrich, St. Louis, $\mathrm{MO}$, USA) diluted in 1X E3 medium, snap-frozen in liquid nitrogen and stored at $-80^{\circ} \mathrm{C}$ prior to DNA extraction. Genomic DNA (gDNA) was extracted from 24hpf embryos using QIAGEN DNeasy Blood \& Tissue Kit (QIAGEN) according to manufacturer's instructions in two biological replicates. 


\section{EM-seq library preparation and sequencing}

EM-seq library construction was performed using the NEBNext Enzymatic Methyl-seq Kit (New England BioLabs, Ipswich, MA, USA) according to manufacturer instructions with modifications. 0.02ng of unmethylated lambda phage DNA (Promega, Madison, WI, USA) and 0.0001 ng of pUC19 plasmid methylated at $100 \%$ of CpG sites (New England BioLabs, Ipswich, MA, USA) were used as spike-in controls to determine the efficiency of APOBEC deamination and TET2 oxidation, respectively. Briefly, 200ng of zebrafish gDNA was sonicated to an average insert size of $300 \mathrm{bp}$. Input DNA concentration was selected according to the optimal input amount as recommended by the manufacturer. Sonicated DNA was end repaired followed by ligation of adapters to DNA overnight using NEXTFLEX Bisulfite-Seq Barcodes (PerkinElmer, Waltham, MA, USA). DNA was treated with TET2 for one hour. Following TET2 oxidation DNA was denatured with $0.1 \mathrm{M} \mathrm{NaOH}$ then treated with APOBEC for three hours. DNA was PCR amplified (8 cycles). Library concentration was quantified through qPCR using KAPA Library Quantification Kit (Sigma-Aldrich, St. Louis, MO, USA) as per manufacturer's instructions. $150 \mathrm{pmol}$ of the combined libraries with 15\% PhiX spike-in was sequenced on the Illumina HiSeq X platform using 150bp paired-end sequencing (high output mode).

\section{WGBS and EM-seq data analyses}

Bisulfite-converted (WGBS) and APOBEC-converted (EM-seq) [14] sequence reads were trimmed with Trimmomatic (ILLUMINACLIP:TruSeq3-SE.fa:2:30:10 SLIDINGWINDOW:5:20 LEADING:3 TRAILING:3 MINLEN:20) [42], and mapped using WALT (-m 5 -t 20 -N 10000000) [43] onto the bisulfite-converted GRCz11 reference (UCSC) containing $\lambda$ (WGBS and EM-seq) and pUC19 sequences (EM-seq) added as separate chromosomes. The resulting alignments in BAM format were processed with CGmapTools [43,44] (convert bam2cgmap) to obtain methylation calls. ATCGmap files were parsed to discard the $\mathrm{CH}$ positions that showed evidence of a $\mathrm{CG}$ position in the reads discordant with the reference genome $\mathrm{CH}$ annotation [11]. Genomic data were visualized in UCSC [45] and IGV [46] browsers.

\section{DNA sequence motif analyses}

Genotype-corrected CGmap files were filtered for $\mathrm{CH}$ positions covered by at least 10 reads, and sorted by methylation level $(\mathrm{mC} / \mathrm{C})$. Top 10,000 positions were then extracted from the reference genome using BEDTools [47] taking the flanking upstream and downstream base pairs $(n=5)$, and preserving the strandedness information. The resulting fasta file was used as input for HOMER "findMotifs.pl" function [48], establishing the search for de novo (-S 5) motifs of length 8 (-len 8) with the default scrambled background option. Motifs were visualized using the "ggseqlogo" package in $R$ [49]. The motif matrix (CATGCTAA) was constructed using the seq2profile.pl HOMER 
function (seq2profile.pl CATGCTAA 0 ets) and the genome-wide motif search was conducted using the scanMotifGenomeWide.pl function (with and without -mask option checked) to uncover CATGCTAA motifs in both repetitive- and non-repetitive DNA.

\section{$\mathrm{mCH}$ level calculation and plotting}

DNA methylation $(\mathrm{mCH})$ levels at CATGCTAA motifs were calculated using BEDtools [47] (map function, -o sum) by dividing the sum of reads supporting a methylated $\mathrm{CH}$ cytosine with the sum of all reads mapping to that position. $\mathrm{mCH}$ levels were plotted using the boxplot function in R (www.r-project.org) (outline = FALSE, notch = TRUE), for positions that had an $\mathrm{mCH}$ value $>0$. Bedgraphs were generated from the corrected CGmap tools output and converted to bigWig using bedGraphToBigwig script from Kent utils (https://github.com/bowhan/kent/tree/master/src/utils). Heatmaps were generated using deepTools [50] computeMatrix and plotHeatmap functions. For WGBS and EMseq data comparisons the heatmaps were generated with the following parameters: "computeMatrix reference-point -b 1500 -a 1500 -p 4 -bs 25, --missingDataAsZero" whereas for plotting of $\mathrm{mCH}$ levels over MOSAT_DR repeats, we used: "computeMatrix scale-regions -m 650 -b 500 -a 500 -p 4 -bs 25 -p 4" with replacement of NAN values with 0 after the matrix file was generated. For profiles (represented as centered heatmaps) the matrices were generated with computeMatrix (computeMatrix referencepoint --referencePoint center -b 1500 -a 1500 -p 4 -bs 50) and plotProfile functions (-plotType heatmap --yMin 0 --yMax 0.15 --perGroup).

\section{Assessment of $\mathrm{mCH}$ in gene bodies}

Zebrafish gene models (ENSEMBL Genes 99, GRCz11) were obtained from www.ensembl.org using the BioMart tool. DNA methylation $(\mathrm{mCH})$ levels in gene bodies were calculated using BEDtools [47] (map function, -o sum) and the number of CATGCTAA motifs in genes was obtained with coverageBed function. Scatterplots of $\mathrm{mCH}$ levels and CATGCTAA motif numbers were generated using the geom_bin2d function in ggplot2 ((bins $=50)+$ geom_smooth $($ method $=\operatorname{lm}))$.

\section{Repeatmasker track analyses}

Repeatmsker track file corresponding to GRCz11 genome reference was downloaded from UCSC. The percentage of repeat subfamilies overlapping CATGCTAA motifs was determined with BEDtools (intersectBed). The genomic annotation of MOSAT_DR motifs was carried out with HOMER [48], annotatePeaks.pl.

\section{Colocalization of histone modification and $\mathrm{mCH}$}

ChIP-seq data sequence reads were trimmed with Trimmomatic (ILLUMINACLIP:TruSeq3-SE.fa:2:30:10 SLIDINGWINDOW:5:20 LEADING:3 TRAILING:3 MINLEN:20) [42], and mapped to GRCz11 genome using bowtie2 default 
settings [51], allowing multi-mapping reads to align to a single (best) genomic location. The resulting alignments in BAM format were deduplicated using sambamba markdup, default settings [52]. RPKM bigWigs were made using deepTools bamCoverage and reads were centered and extended by 300 base pairs (-e 300 -p 20 --normalizeUsing RPKM --centerReads) [50]. For H3K9me3 data sets, where input data was available, subtraction of input signal was also performed using deepTools bigWigCompare (-operation subtract) before heatmaps were plotted [50]. Heatmaps of histone RPKM levels over MOSAT_DR elements were generated using deepTools computeMatrix (computeMatrix scale-regions -m 650 -b 500 -a 500 -p 4 -bs 25 -p 4), with NAN values replaced with 0 after completion, and were visualized with sorting based on the $\mathrm{mCH}$ datasets (plotHeatmap --sortUsingSamples). Data for scatterplots were generated using bedtools map, to determine average $\mathrm{mCH}$ levels, and bedtools intersect (-abam), bedtools intersect $(-c)$ and samtools flagstat, to calculate H3K9me3 RPKM $[47,53]$. Scatterplots were generated using the geom_bin2d function in ggplot2 ((bins $=75)+$ geom_smooth(method=Im) and Pearson's correlation were determined by the rcorr function in $\mathrm{R}$. 


\section{References}

1. Schubeler D. Function and information content of DNA methylation. Nature. 2015;517:321-6.

2. de Mendoza A, Lister R, Bogdanovic O. Evolution of DNA Methylome Diversity in Eukaryotes. J Mol Biol. 2019;432:1687-1705.

3. Lister R, Pelizzola M, Dowen RH, Hawkins RD, Hon G, Tonti-Filippini J, et al. Human DNA methylomes at base resolution show widespread epigenomic differences. Nature. 2009;462:315-22.

4. Lister R, Mukamel EA, Nery JR, Urich M, Puddifoot CA, Johnson ND, et al. Global epigenomic reconfiguration during mammalian brain development. Science.

2013;341:1237905.

5. Schultz MD, He Y, Whitaker JW, Hariharan M, Mukamel EA, Leung D, et al. Human body epigenome maps reveal noncanonical DNA methylation variation. Nature. 2015;523:212-216.

6. Arand J, Spieler D, Karius T, Branco MR, Meilinger D, Meissner A, et al. In vivo control of $\mathrm{CpG}$ and non-CpG DNA methylation by DNA methyltransferases. PLoS Genet. 2012;8:e1002750.

7. Luo C, Lee QY, Wapinski O, Castanon R, Nery JR, Mall M, et al. Global DNA methylation remodeling during direct reprogramming of fibroblasts to neurons. Elife. 2019;8.

8. Lister R, Pelizzola M, Kida YS, Hawkins RD, Nery JR, Hon G, et al. Hotspots of aberrant epigenomic reprogramming in human induced pluripotent stem cells. Nature. 2011;471:68-73.

9. Ziller MJ, Muller F, Liao J, Zhang Y, Gu H, Bock C, et al. Genomic distribution and inter-sample variation of non-CpG methylation across human cell types. PLoS Genet. 2011;7:e1002389.

10. He Y, Ecker JR. Non-CG Methylation in the Human Genome. Annual Review of Genomics and Human Genetics. 2015;55-77.

11. de Mendoza A, Popper D, Buckberry S, Pflueger J, Albertin C, Daish T, et al. The emergence of neural non-CpG methylation system in vertebrates. Under review. 2020.

12. Bogdanovic O, Smits AH, de la Calle Mustienes E, Tena JJ, Ford E, Williams R, et al. Active DNA demethylation at enhancers during the vertebrate phylotypic period. Nat Genet. 2016;48:417-26. https://doi.org/10.1038/ng.3522.

13. Olova N, Krueger F, Andrews S, Oxley D, Berrens RV, Branco MR, et al. Comparison of whole-genome bisulfite sequencing library preparation strategies 
identifies sources of biases affecting DNA methylation data. Genome Biol. 2018;19:33.

14. Vaisvila R, Chaithanya Ponnaluri VK, Sun Z, Langhorst BW, Saleh L, Guan S, et al. EM-seq: Detection of DNA Methylation at Single Base Resolution from Picograms of DNA. BioRxiv. Available from: http://dx.doi.org/10.1101/2019.12.20.884692

15. Raudvere U, Kolberg L, Kuzmin I, Arak T, Adler P, Peterson H, et al. g:Profiler: a web server for functional enrichment analysis and conversions of gene lists (2019 update). Nucleic Acids Research. 2019;191-8.

16. Gabel HW, Kinde B, Stroud H, Gilbert CS, Harmin DA, Kastan NR, et al. Disruption of DNA-methylation-dependent long gene repression in Rett syndrome. Nature. 2015;522:89-93.

17. Boxer LD, Renthal W, Greben AW, Whitwam T, Silberfeld A, Stroud H, et al. MeCP2 Represses the Rate of Transcriptional Initiation of Highly Methylated Long Genes. Mol Cell. 2020;77:294-309.e9.

18. Thisse B, Thisse C. Fast Release Clones: A High Throughput Expression Analysis. ZFIN Direct Data Submission. http://zfin.org. Accessed on , 2020.

19. Andersen IS, Reiner AH, Aanes H, Alestrom P, Collas P. Developmental features of DNA methylation during activation of the embryonic zebrafish genome. Genome Biol. 2012;13:R65.

20. Potok ME, Nix DA, Parnell TJ, Cairns BR. Reprogramming the maternal zebrafish genome after fertilization to match the paternal methylation pattern. Cell. 2013;153:75972.

21. Jiang L, Zhang J, Wang J-J, Wang L, Zhang L, Li G, et al. Sperm, but Not Oocyte, DNA Methylome Is Inherited by Zebrafish Early Embryos. Cell. 2013;153:773-84. doi: 10.1016/j.cell.2013.04.041.

22. McGaughey DM, Abaan HO, Miller RM, Kropp PA, Brody LC. Genomics of CpG methylation in developing and developed zebrafish. G3. 2014;4:861-9.

23. Mustienes E de la C, de la Calle Mustienes E, Gómez-Skarmeta JL, Bogdanović O. Genome-wide epigenetic cross-talk between DNA methylation and H3K27me3 in zebrafish embryos. Genomics Data. 2015;7-9.

24. Skvortsova K, Tarbashevich K, Stehling M, Lister R, Irimia M, Raz E, et al. Retention of paternal DNA methylome in the developing zebrafish germline. Nat Commun. 2019; 10:3054. https://doi.org/10.1038/s41467-019-10895-6

25. Ortega-Recalde O, Day RC, Gemmell NJ, Hore TA. Zebrafish preserve global germline DNA methylation while sex-linked rDNA is amplified and demethylated during feminisation. Nat Commun. 2019;10:3053. 
26. Murphy PJ, Wu SF, James CR, Wike CL, Cairns BR. Placeholder Nucleosomes Underlie Germline-to-Embryo DNA Methylation Reprogramming. Cell. 2018;172:9931006.

27. Lee HJ, Lowdon RF, Maricque B, Zhang B, Stevens M, Li D, et al. Developmental enhancers revealed by extensive DNA methylome maps of zebrafish early embryos. Nature Commun. 2015;6:6315

28. White RJ, Collins JE, Sealy IM, Wali N, Dooley CM, Digby Z, et al. A high-resolution mRNA expression time course of embryonic development in zebrafish. Elife. 2017;6. https://doi.org/10.7554/eLife.30860

29. Liu J, Hu H, Panserat S, Marandel L. Evolutionary history of DNA methylation related genes in chordates: new insights from multiple whole genome duplications. Sci Rep. 2020;10:970. https://doi.org/10.1038/s41598-020-57753-w

30. Smith ZD, Chan MM, Mikkelsen TS, Gu H, Gnirke A, Regev A, et al. A unique regulatory phase of DNA methylation in the early mammalian embryo. Nature. 2012;484:339-44.

31. Guo H, Zhu P, Yan L, Li R, Hu B, Lian Y, et al. The DNA methylation landscape of human early embryos. Nature. 2014;511:606-10.

32. Smith ZD, Chan MM, Humm KC, Karnik R, Mekhoubad S, Regev A, et al. DNA methylation dynamics of the human preimplantation embryo. Nature. 2014;511:611-5.

33. Lee HJ, Hore TA, Reik W. Reprogramming the Methylome: Erasing Memory and Creating Diversity. Cell Stem Cell. 2014;710-9.

34. Wang L, Zhang J, Duan J, Gao X, Zhu W, Lu X, et al. Programming and inheritance of parental DNA methylomes in mammals. Cell. 2014;157:979-91.

35. Laue K, Rajshekar S, Courtney AJ, Lewis ZA, Goll MG. The maternal to zygotic transition regulates genome-wide heterochromatin establishment in the zebrafish embryo. Nat Commun. 2019;10:1551. https://doi.org/10.1038/s41467-019-09582-3.

36. Bogdanovic O, Fernandez-Miñán A, Tena JJ, de la Calle-Mustienes E, Hidalgo C, van Kruysbergen I, et al. Dynamics of enhancer chromatin signatures mark the transition from pluripotency to cell specification during embryogenesis. Genome Res. 2012;22:2043-53. https://dx.doi.org/10.1101\%2Fgr.134833.111

37. Zhang B, Wu X, Zhang W, Shen W, Sun Q, Liu K, et al. Widespread Enhancer Dememorization and Promoter Priming during Parental-to-Zygotic Transition. Mol Cell. 2018;72:673-686. https://doi.org/10.1016/j.molcel.2018.10.017

38. Lindeman LC, Andersen IS, Reiner AH, Li N, Aanes H, Østrup O, et al. Prepatterning of Developmental Gene Expression by Modified Histones before Zygotic Genome Activation. Developmental Cell. 2011;13:993-1004. 
39. Collings CK, Anderson JN. Links between DNA methylation and nucleosome occupancy in the human genome. Epigenetics Chromatin. 2017;10:18.

40. Lehnertz B, Ueda Y, Derijck AAHA, Braunschweig U, Perez-Burgos L, Kubicek S, et al. Suv39h-mediated histone H3 lysine 9 methylation directs DNA methylation to major satellite repeats at pericentric heterochromatin. Curr Biol. 2003;13:1192-200.

41. Rai K, Jafri IF, Chidester S, James SR, Karpf AR, Cairns BR, et al. Dnmt3 and G9a cooperate for tissue-specific development in zebrafish. J Biol Chem. 2010;285:4110-21.

42. Bolger AM, Lohse M, Usadel B. Trimmomatic: a flexible trimmer for Illumina sequence data. Bioinformatics. 2014;30:2114-20.

43. Chen $\mathrm{H}$, Smith $A D$, Chen $T$. WALT: fast and accurate read mapping for bisulfite sequencing. Bioinformatics. 2016;32:3507-9.

44. Guo W, Zhu P, Pellegrini M, Zhang MQ, Wang X, Ni Z. CGmapTools improves the precision of heterozygous SNV calls and supports allele-specific methylation detection and visualization in bisulfite-sequencing data. Bioinformatics. 2018;34:381-7.

45. Kent WJ, Sugnet CW, Furey TS, Roskin KM, Pringle TH, Zahler AM, et al. The human genome browser at UCSC. Genome Res. 2002;12:996-1006.

46. Robinson $P$, Jtel TZ. Integrative genomics viewer (IGV): Visualizing alignments and variants. Computational Exome and Genome Analysis. 2017; 233-45.

47. Quinlan AR, Hall IM. BEDTools: a flexible suite of utilities for comparing genomic features. Bioinformatics. 2010;26:841-2.

48. Heinz S, Benner C, Spann N, Bertolino E, Lin YC, Laslo P, et al. Simple Combinations of Lineage-Determining Transcription Factors Prime cis-Regulatory Elements Required for Macrophage and B Cell Identities. Molecular Cell. 2010;38:57689.

49. Wagih O. ggseqlogo: a versatile $R$ package for drawing sequence logos. Bioinformatics. 2017;33:3645-7.

50. Ramirez F, Dundar F, Diehl S, Gruning BA, Manke T. deepTools: a flexible platform for exploring deep-sequencing data. Nucleic Acids Res. 2014;42:187-91.

51. Langmead B, Salzberg SL. Fast gapped-read alignment with Bowtie 2. Nat Methods. 2012;9:357-9.

52. Tarasov A, Vilella AJ, Cuppen E, Nijman IJ, Prins P. Sambamba: fast processing of NGS alignment formats. Bioinformatics. 2015;31:2032-4.

53. Li H, Handsaker B, Wysoker A, Fennell T, Ruan J, Homer N, et al. The Sequence Alignment/Map format and SAMtools. Bioinformatics. 2009;25:2078-9. 


\section{Competing interests}

The authors declare that they have no competing interests.

\section{Funding}

Australian Research Council (ARC) Discovery Project (DP190103852) to OB supported this work. OB is supported by NHMRC (R.D. Wright Biomedical CDF APP1162993) and CINSW (Career Development Fellowship CDF181229).

\section{Author contribution}

OB conceived the study. SR, OB, and AdM performed bioinformatic analyses. SR and OB wrote the paper. AA performed EM-seq experiments. All authors contributed to, read, and approved the final manuscript.

\section{Acknowledgments}

We thank Michael Geng for the management and breeding of zebrafish colonies used in EM-seq libraries and Ksenia Skvortsova for input into EM-seq experiments. We thank Ryan Lister for input on $\mathrm{mCH}$ data analysis and for critical comments on the manuscript. We thank Sriharsa Pradhan and New England Biolabs for assistance with the EM-seq kit. Images of zebrafish embryos and cell types were created with Biorender.

\section{Availability of data and materials}

Data generated for this submission have been uploaded to the Gene Expression Omnibus (GEO) (https://www.ncbi.nlm.nih.gov/geo) under the record: GSE149416. The following secure token has been created to allow review while this submission remains in private status: cbmlqggctrixzip. WGBS datasets analysed in this study were taken from the following GEO records: \%80 epiboly (GSM1662779), 24hpf (GSM1662780), 48hpf (GSM1662781) and adult brain (GSM1662792) (https://www.ncbi.nlm.nih.gov/geo/query/acc.cgi?acc=GSE68087) [12]. Egg (GSM1133392), sperm (GSM1133391), 32cell (GSM1133394), 64cell (GSM1133395) and germring (GSM1133398) (https://www.ncbi.nlm.nih.gov/geo/query/acc.cgi?acc=GSE44075) [21]. 4hpf (GSM3484060, GSM3484068), 7hpf (GSM3484061, GSM3484069) and liver (GSM3505059) (https://www.ncbi.nlm.nih.gov/geo/query/acc.cgi?acc=GSE122722) [24]. ChIP seq datasets analysed in this study were taken from the following GEO records: H3K9me3 at 6hpf (GSM3096185, GSM3096186), 4.5hpf (GSM3096189, GSM3096190) and 2.5hpf (GSM3096193, GSM3096194)

(https://www.ncbi.nlm.nih.gov/geo/query/acc.cgi?acc=GSE113086) [35]. H3K27me3 (GSM3165233) and H3K36me3 (GSM3165232) at dome stage (https://www.ncbi.nlm.nih.gov/geo/query/acc.cgi?acc=GSE114954) [37]. H3K27ac 
(GSM803830), H3K4me1(GSM915193) and H3K4me3 (GSM915190) at dome stage (https://www.ncbi.nlm.nih.gov/geo/query/acc.cgi?acc=GSE32483) [36].

\section{Supplementary information}

Additional file 1: Supplementary figures S1-S5 and Supplementary tables S1-S2 Additional file 2: MicroBam file for visualisation of reads at four genes covered by MOSAT DR elements.

Figure 1 MOSAT motifs are enriched for $\mathrm{mCH}$. a Genomic and lambda control dinucleotide $\% \mathrm{mCH}$ in zebrafish embryos and adult brain. $\mathbf{b}$ Motif analyses of most methylated $\mathrm{CH}$ sites $(\mathrm{n}=10,000)$ in zebrafish embryos and adult brain. $\mathbf{c}$ Boxplots showing average $\mathrm{mCH} / \mathrm{CH}$ levels of the CATGCTAA motif in repeat-masked and repetitive portions of the genome. $\mathbf{d}$ Repetitive element annotation of CATGCTAA motifcontaining regions. e Per read analysis of MOSAT motif $\mathrm{mCH} / \mathrm{CH}$ in MOSAT_DR elements. $\mathbf{f}$ Heatmap of methylated $(\mathrm{mCH} / \mathrm{CH}>0.1)$ MOSAT motifs in $24 \mathrm{hpf}$ embryos identified by WGBS and compared to EM-seq signal and (g) identified by EM-seq and compared to WGBS signal.

Figure $2 \mathrm{mCH}$ at MOSAT_DR elements is developmentally reprogrammed and associated with constitutive $\mathrm{H} 3 \mathrm{~K} 9 \mathrm{me}$. a UCSC browser snapshot of $\mathrm{mCH}$ and $\mathrm{mCG}$ levels in both intronic and intergenic MOSAT_DR elements in developing zebrafish embryos and adult brain. B Scatterplot of average gene methylation $(\mathrm{mCH} / \mathrm{CH})$ plotted against the number of MOSAT motifs in gene bodies of $80 \%$ epiboly zebrafish embryos. c $\mathrm{mCH}$ profiles of MOSAT motifs $(n=8,064)$ in commonly covered MOSAT_DR elements during zebrafish development. d Heatmap of $\mathrm{CH}$ methylation $(\mathrm{mCH} / \mathrm{CH})$ and $\mathrm{H} 3 \mathrm{~K} 9 \mathrm{me} 3$ (RPKM) levels in commonly covered MOSAT_DR elements $(n=3,091)$ during zebrafish development. e UCSC browser snapshot of $\mathrm{mCG}$ and $\mathrm{mCH}$ in $80 \%$ epiboly and $24 \mathrm{hpf}$ embryos compared to H3K9me3 (log2 ChIP/Input) in shield stage and dome embryos. $f$ Scatterplots showing the correlation between $\mathrm{mCH} / \mathrm{CH}$ at various time points and the level of H3K9me3 (log2RPKM) from shield stage embryos. Left to right: 32cell, sphere, $80 \%$ epiboly, $80 \%$ epiboly vs input. 


\section{Figure 1}

a

b

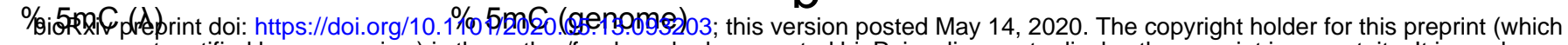
$0.4 \quad 0.2$ not certified by peer review) is the author/funder who has granted bioRxiv a license to display the preprint in perpetuity. It is made

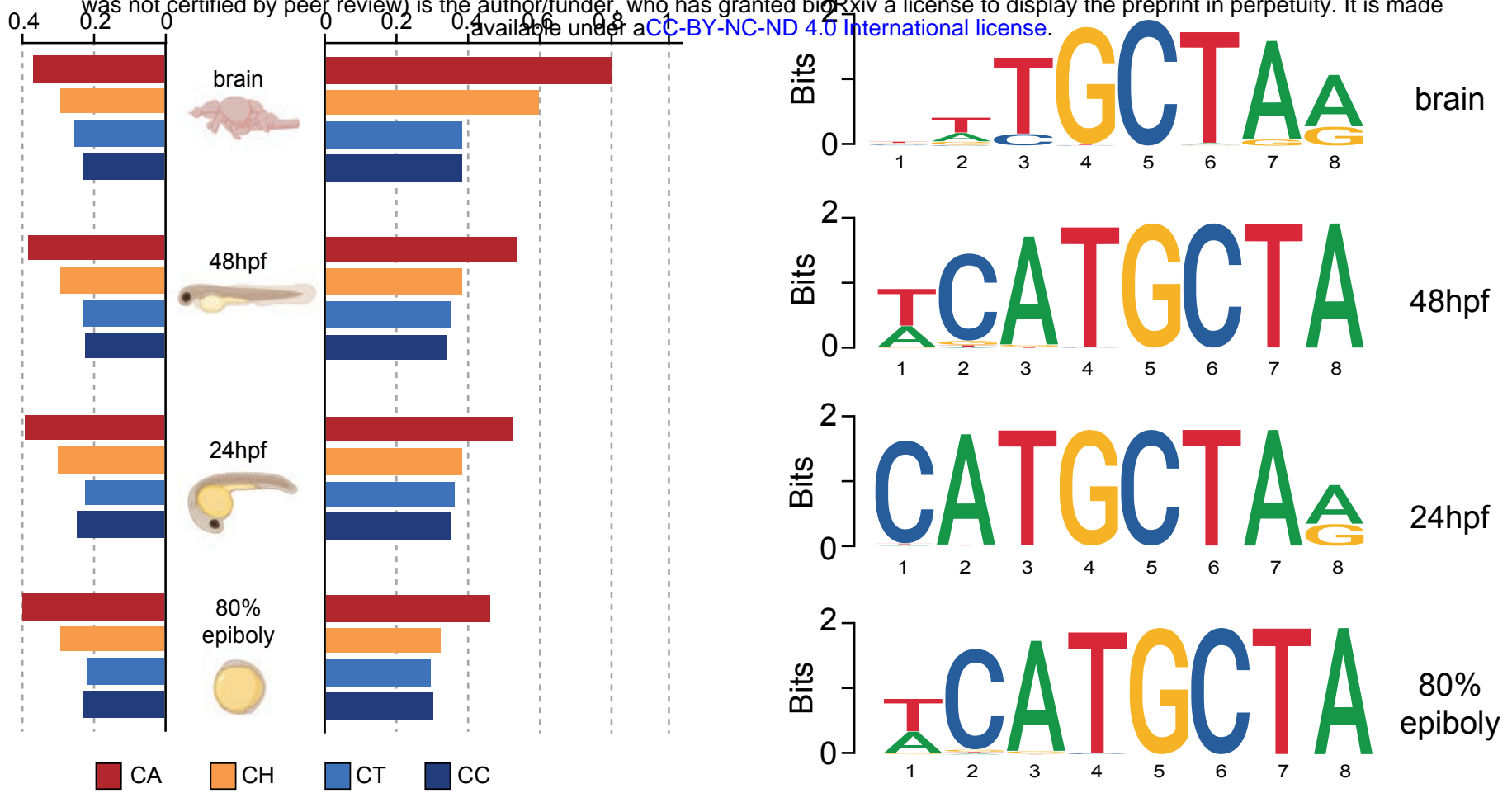

C
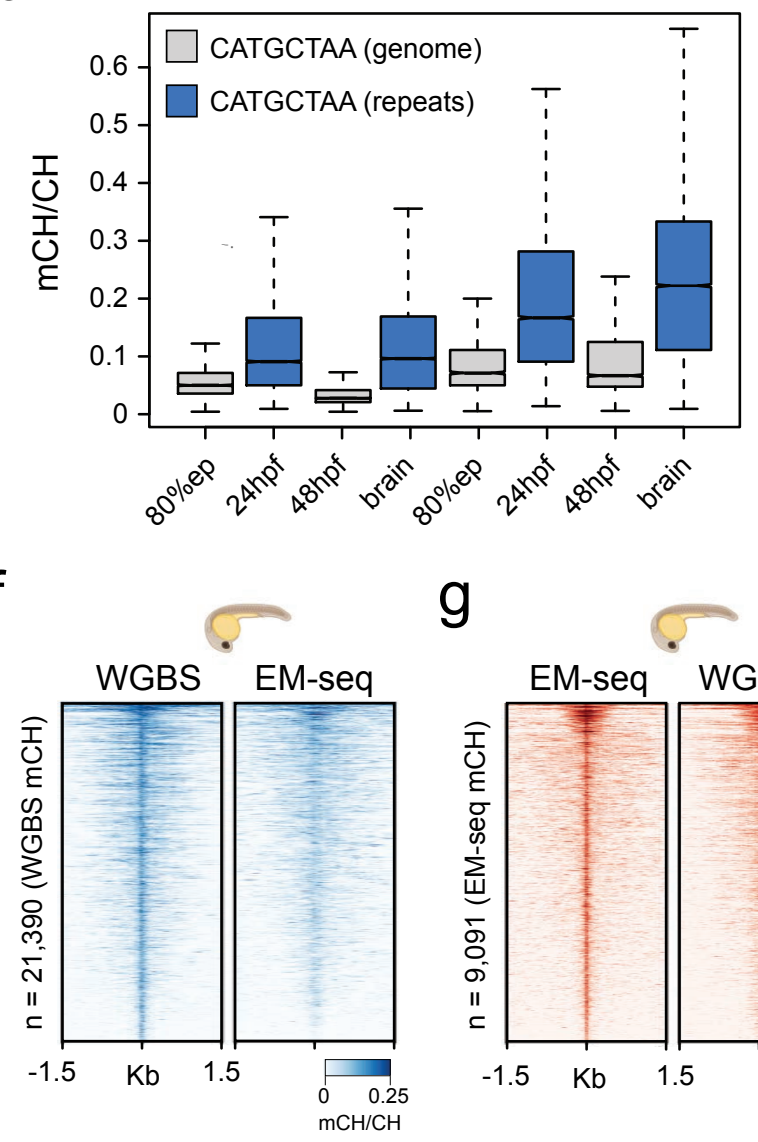

g

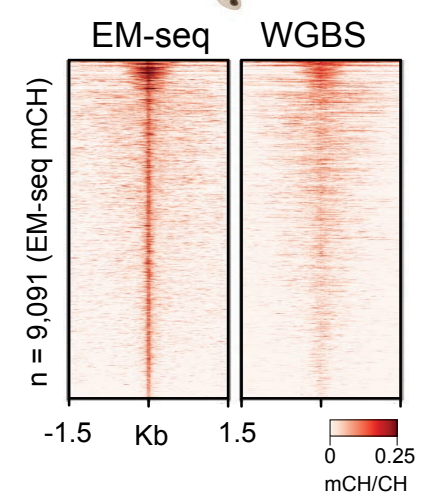

d

e

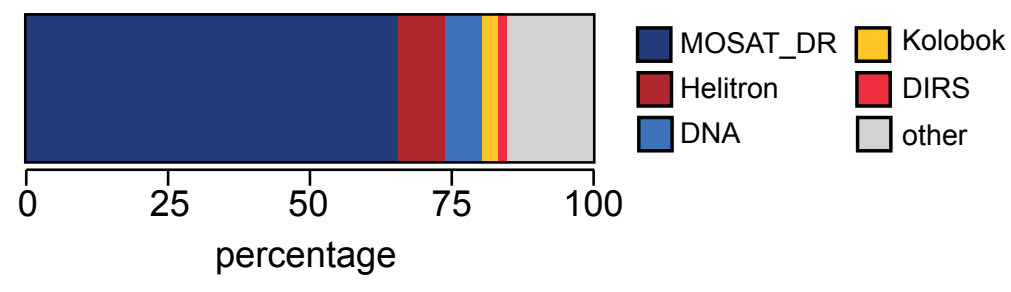

$\mathrm{mCH} / \mathrm{CH}$
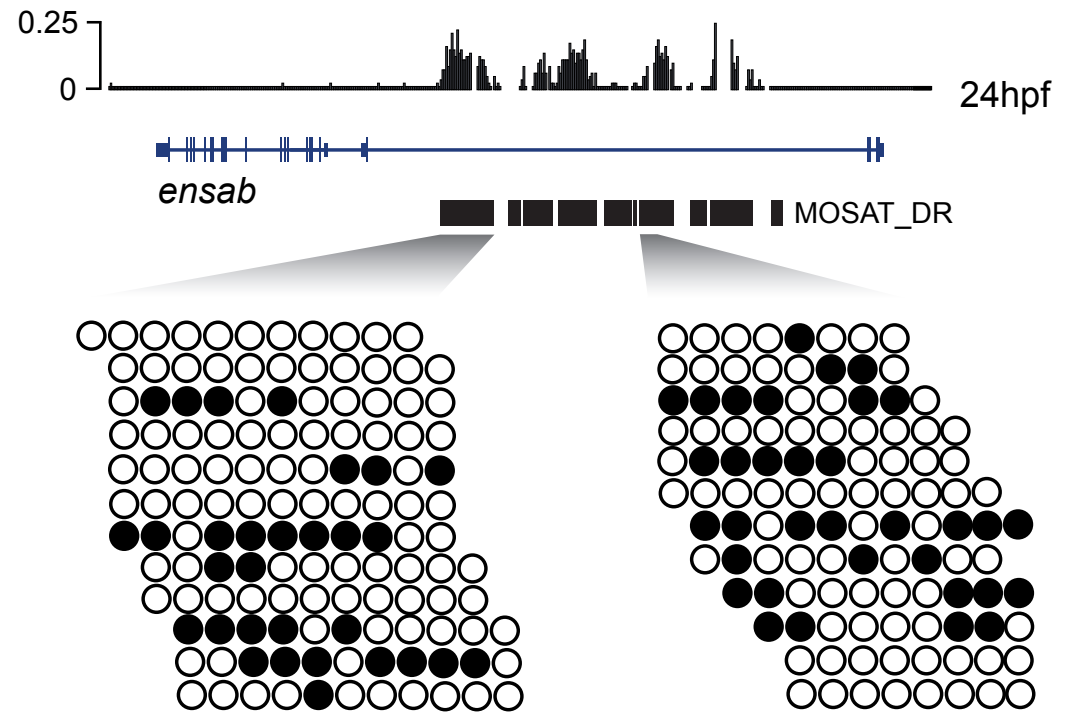

O TGCT

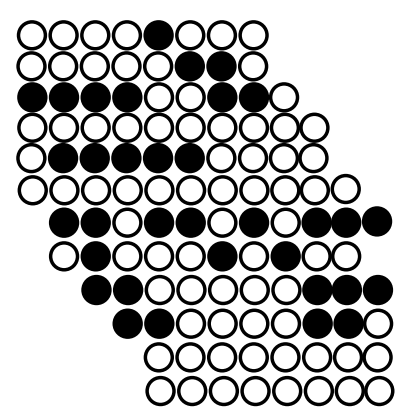

- TGmCT 
_. bioRxiv preprint doi: https://doi.org/10.1101/2020.05.13.093203; this version posted May 14,2020 . The copyright holder for this preprint (which Figurew not certified by peer review) is the author/funder, who has granted bioRxiv a license to display the preprint in perpetuity. It is made available under ACC-BY-NC-ND 4.0 International license.

a
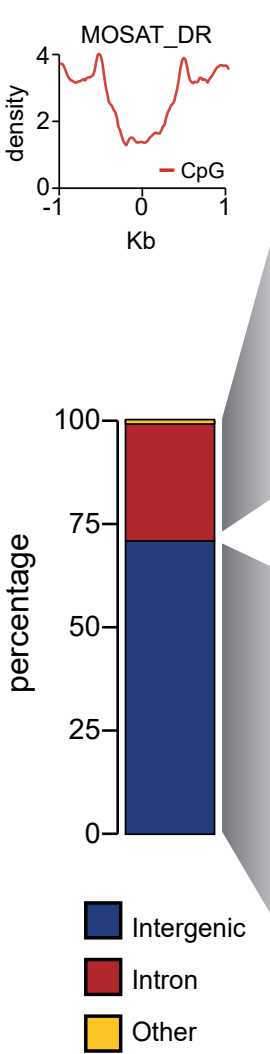

d

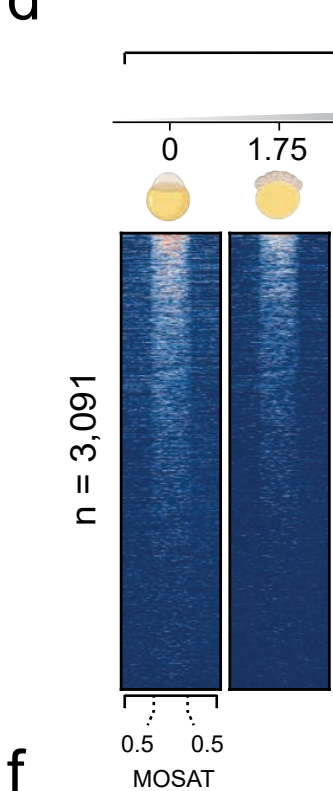

(kb)

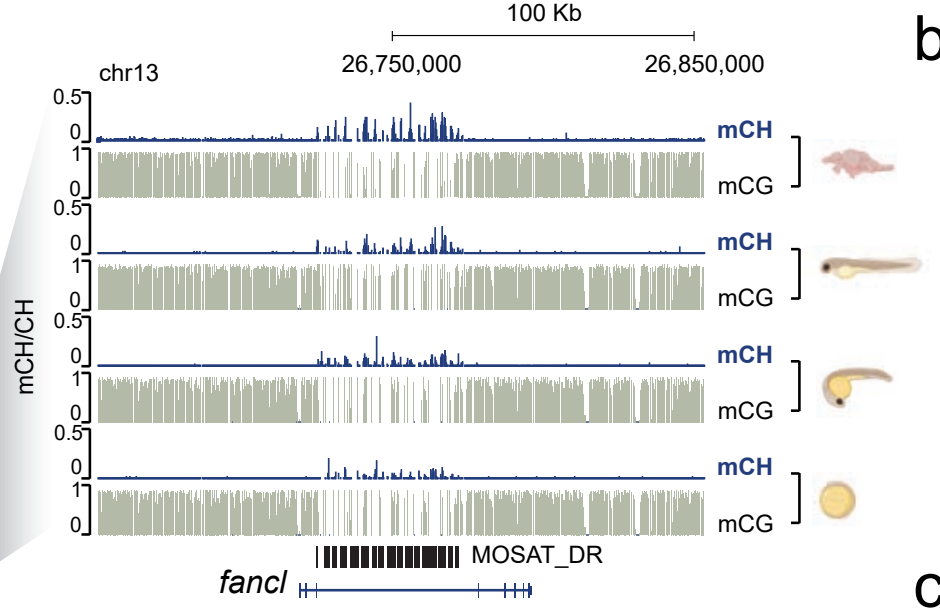

C

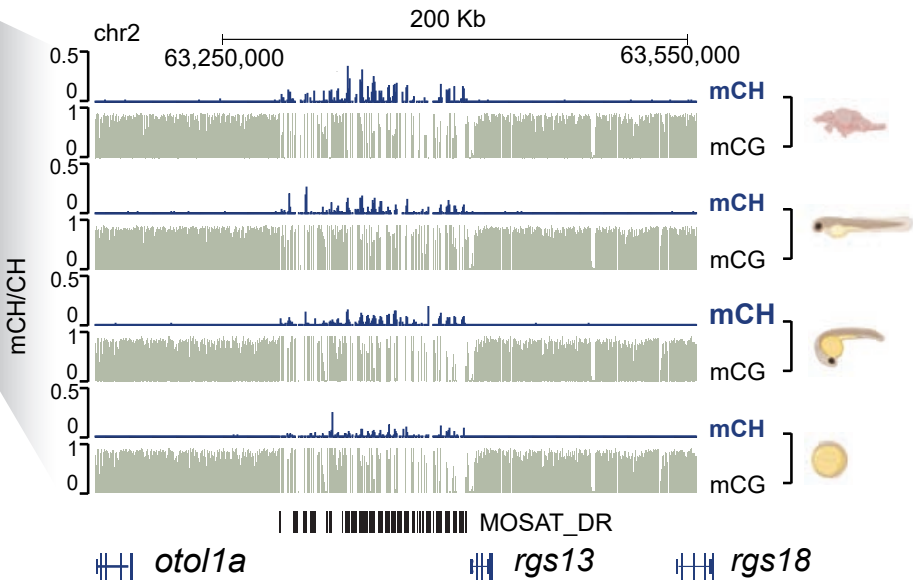

e

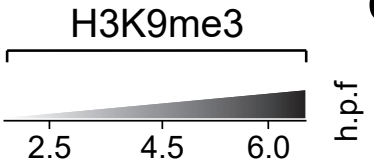

b

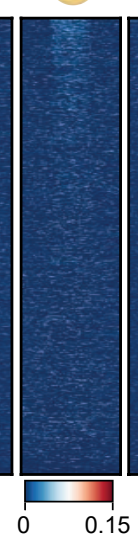

$\mathrm{mCH} / \mathrm{CH}$
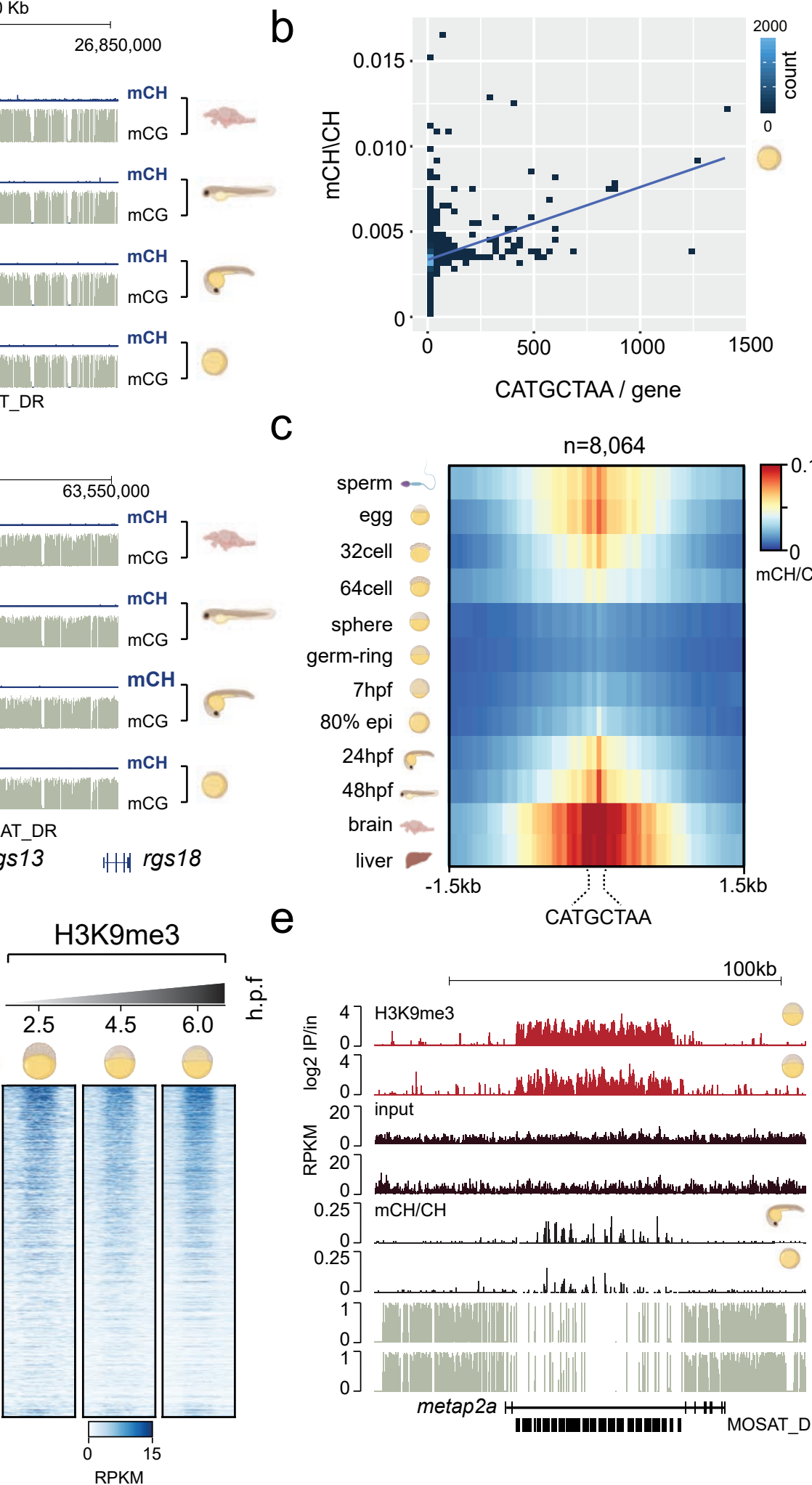

C

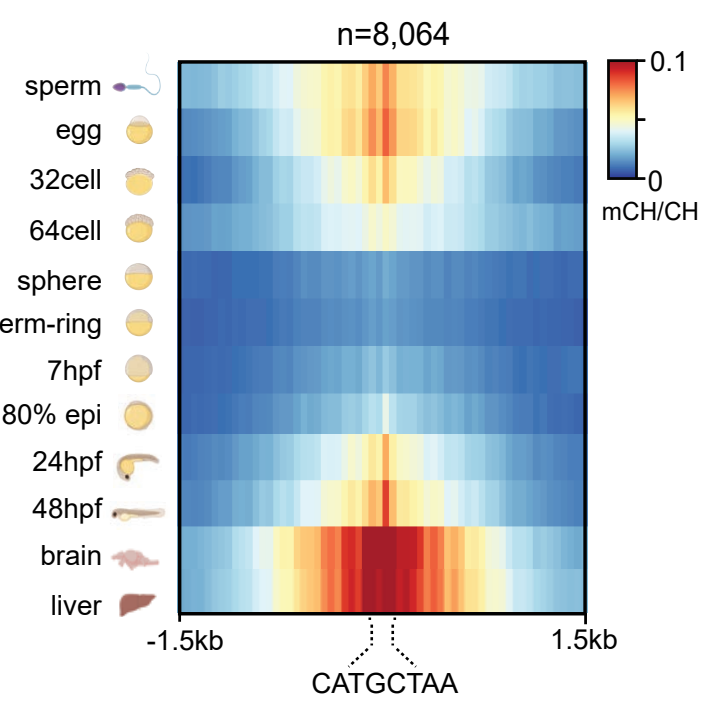

$100 \mathrm{~kb}$

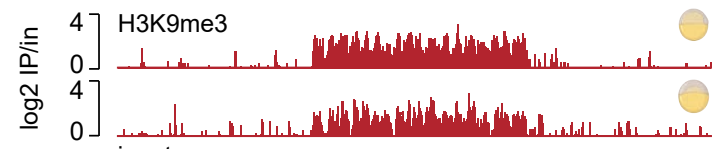

207 input

$\sum_{\substack{\alpha \\ \frac{x}{\alpha}}} 0$

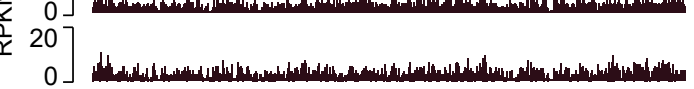

$0.25] \mathrm{mCH} / \mathrm{CH}$

0
0.25

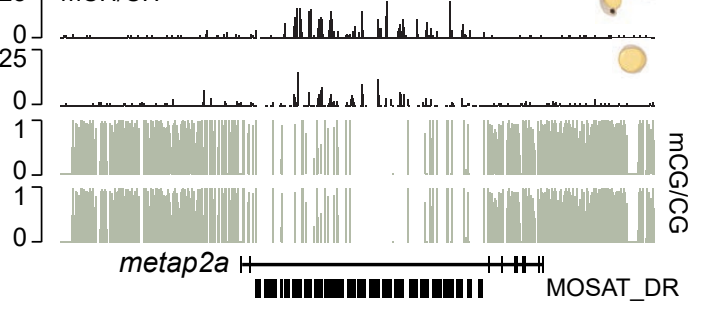

f

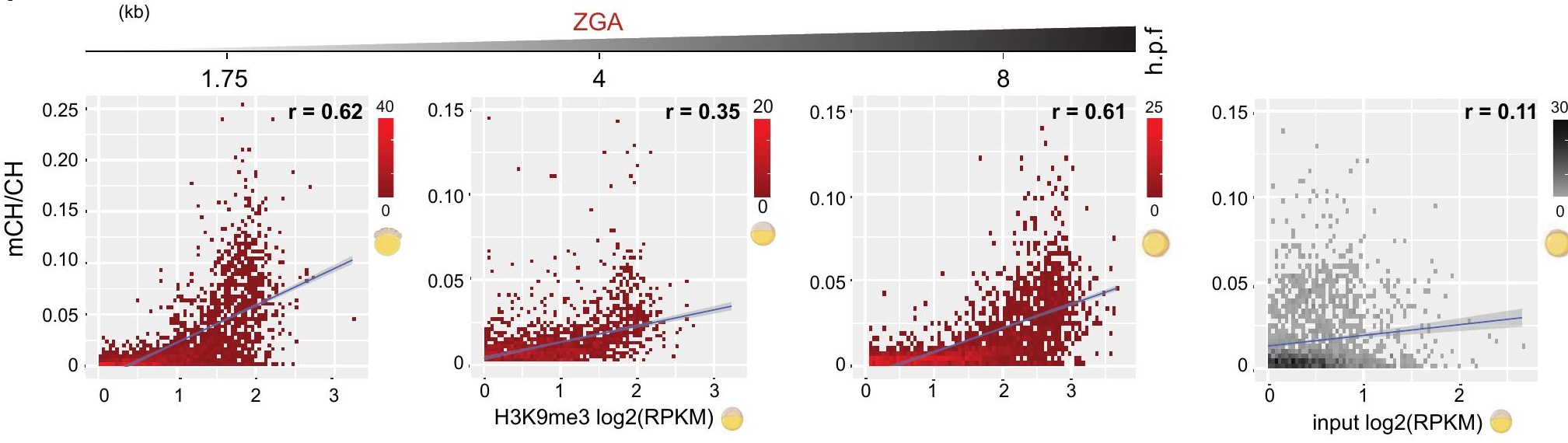



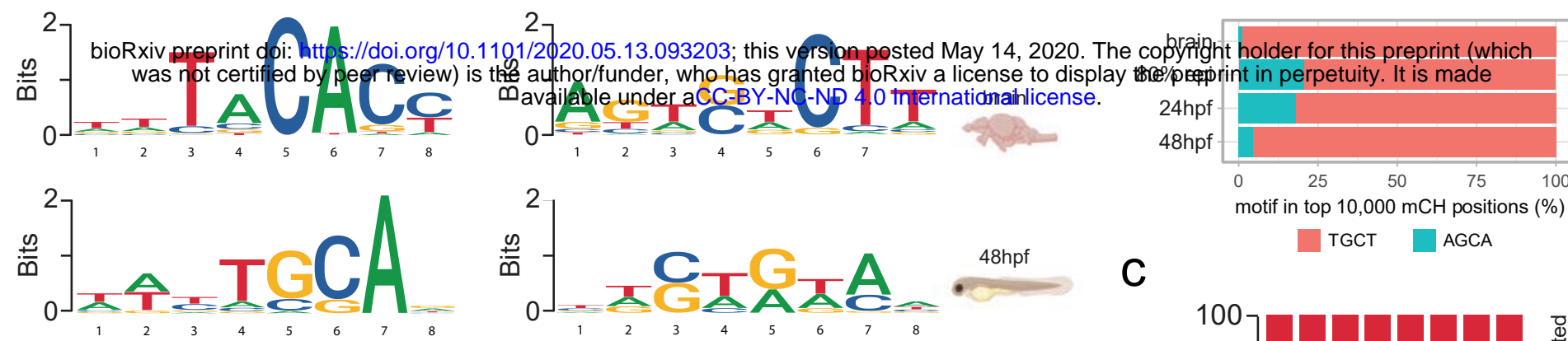

$48 \mathrm{hpf}$

TGCT AGCA
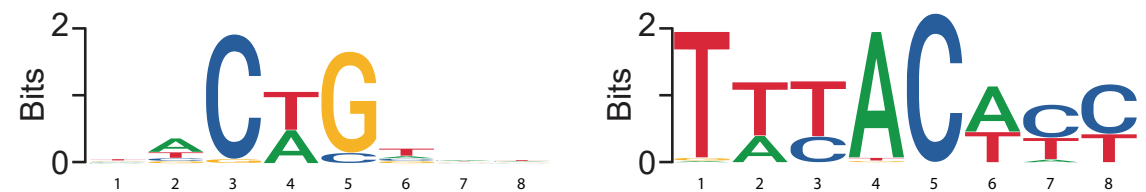

$24 \mathrm{hpf}$
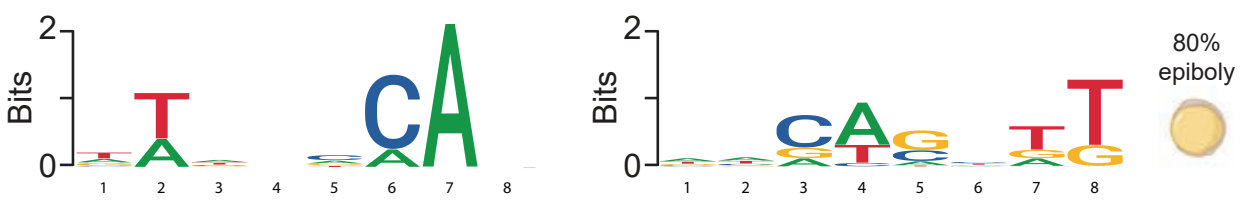

C

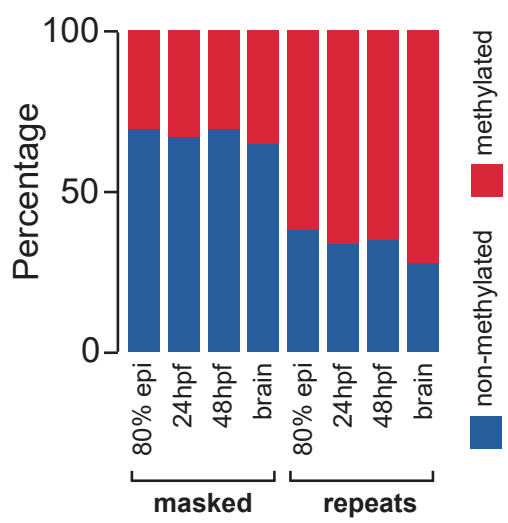

Figure S1. a 2nd and 3rd ranked motifs from motif search conducted on 10,000 most highly methyl ated $\mathrm{CH}$ sites in zebrafish embryos and adult brain. $\mathbf{b}$ Methylation levels of TGCT and AGCA tetranucleotides in 10,000 most highly methylated $\mathrm{CH}$ sites in zebrafish embryos and adult brain. c Percentage of CATGCTAA motifs exhibiting methylation in zebrafish embryos and adult brains in repetitive and repeat-masked portions of the genome 


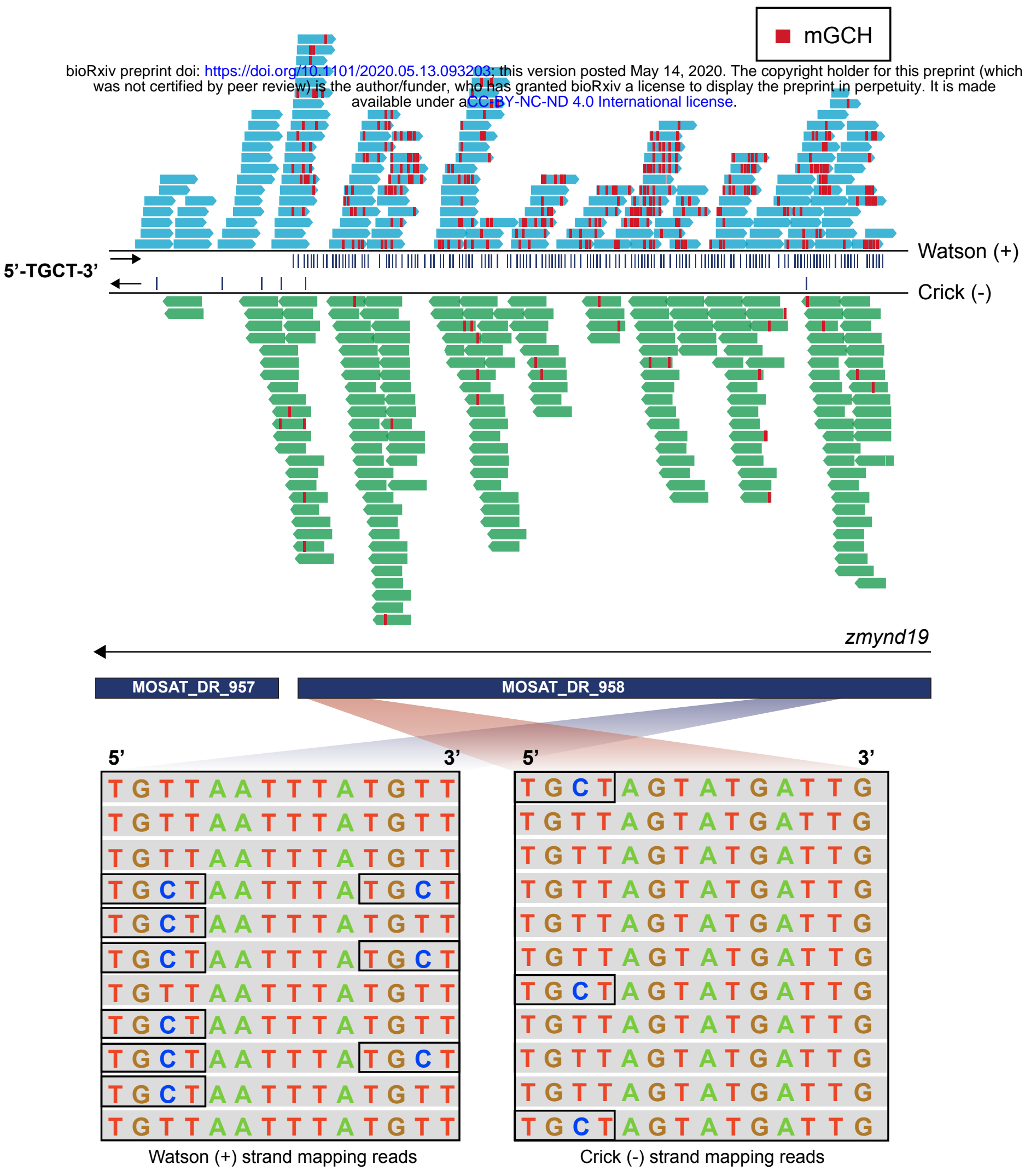

Figure S2. IGV Visualisation of WGBS reads from 24hpf zebrafish embryos mapped with 0 mismatches and aligned to MOSAT_DR elements. Blue reads (forward) map to the Watson(+) strand and contain predominately 5'-TGCT-3' tetranucleotides (blue arrows) while green reads (reverse) map to the Crick (-) strand. Red lines indicate the positions of methylated GCH nucleotides, which are enriched on the Watson (+) strand and coincide with 5'-TGCT-3' tetranucleotides. Boxes contain base-resolution visualisation of forward and reverse reads and highlight methylated 5'-TGCT-3' tetranucleotides within which cytosine did not undergo bisulfite conversion. 


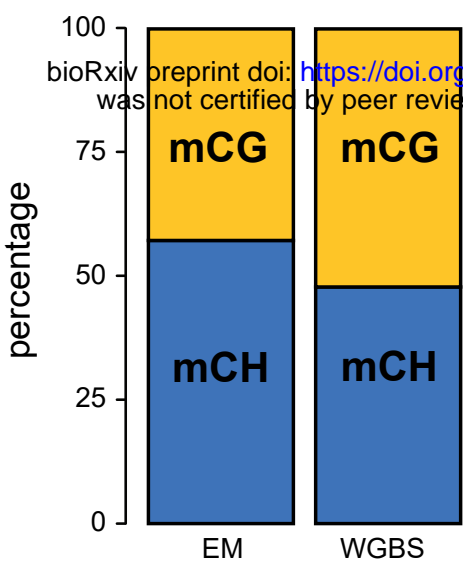

d

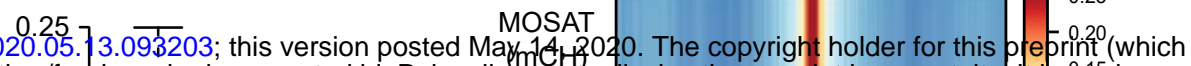
license to display the preprint in perpetuity it is phisde

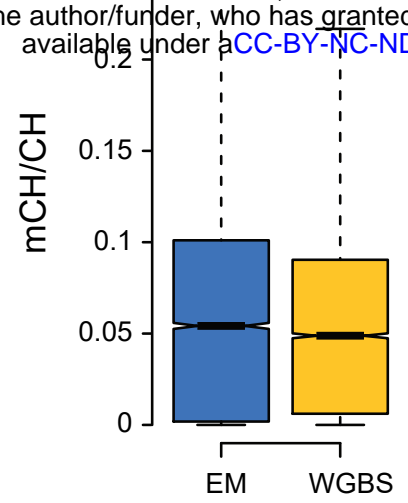
.

(genome)

$-3 K b$

MOSAT (CATGCTAA)

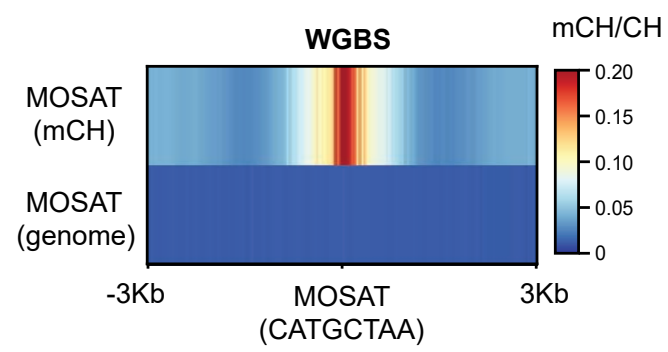

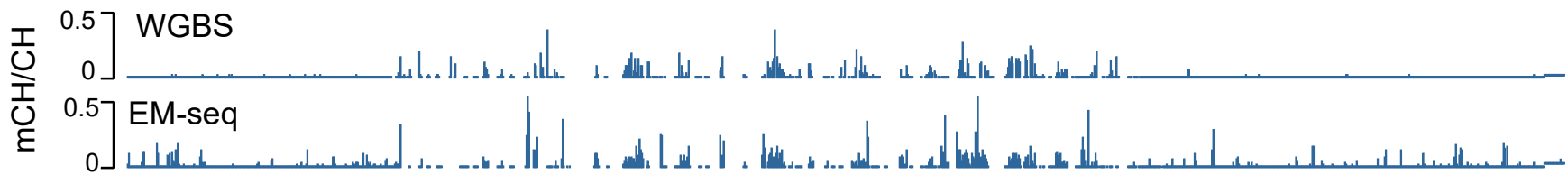

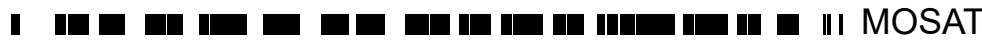

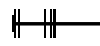

e fancl

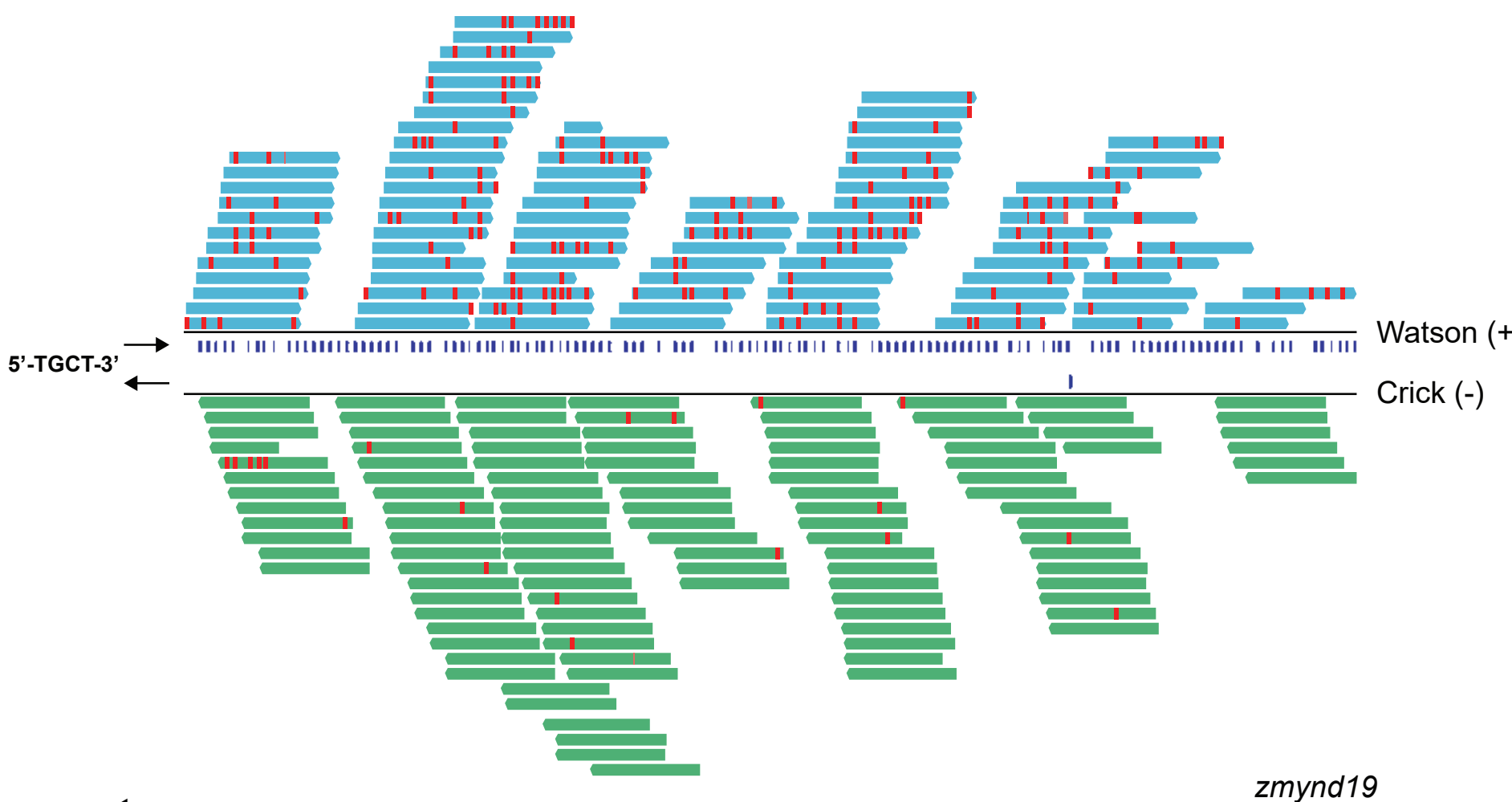

Figure S3. a Proportion of methylated sites $(\geq 0.1 \mathrm{mC} / \mathrm{C})$ in MOSAT DR elements in $\mathrm{CG}$ and $\mathrm{CH}$ contexts determined by EM-seq and WGBS from 24hpf zebrafish embryos. $\mathbf{b}$ Boxplots of average mCH levels of MOSAT DR elements in 24hpf zebrafish embryos determined by EM-seq and WGBS. c Heatmap profiles showing mCH at most highly methylated MOSAT motif sites $(\geq 0.1 \mathrm{mCH} / \mathrm{CH})$ as identified by EM-seq and WGBS in 24hpf zebrafish embryos, and the same number of sites randomly selected from non-repetitive DNA. d UCSC browser snaphshot of $\mathrm{mCH}$ levels in 24hpf zebrafish embryos at the fancl locus, determined by EM-seq and WGBS. e IGV Visualisation of EM-seq reads from 24hpf zebrafish embryos aligned to MOSAT_DR elements in the zmynd19 locus. Blue reads (forward) map to the Watson(+) strand and contain predominately 5'-TGCT-3' tetranucleotides (blue lines) while green reads (reverse) map to the Crick (-) strand. Red lines indicate methylated GCH positions which are enriched on the Watson (+) strand and 5'-TGCT-3' tetranucleotides. 


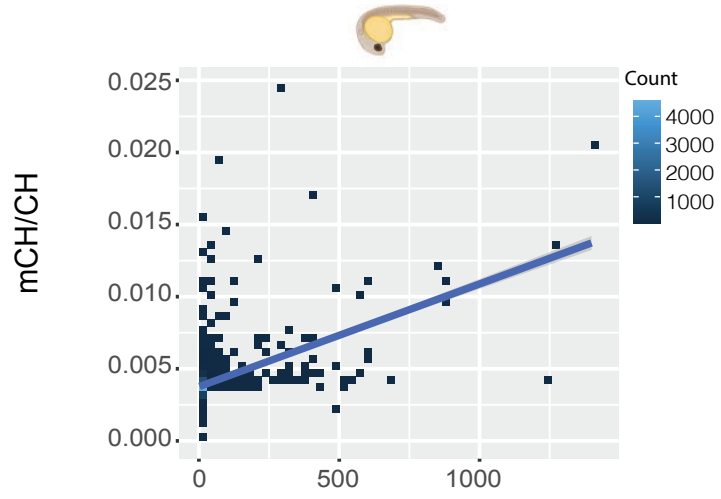

b

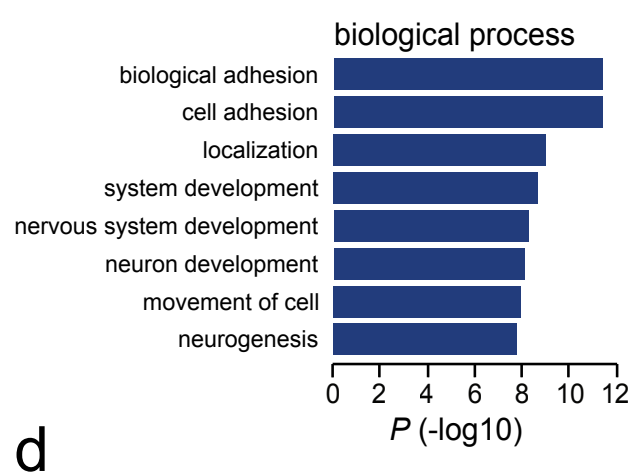

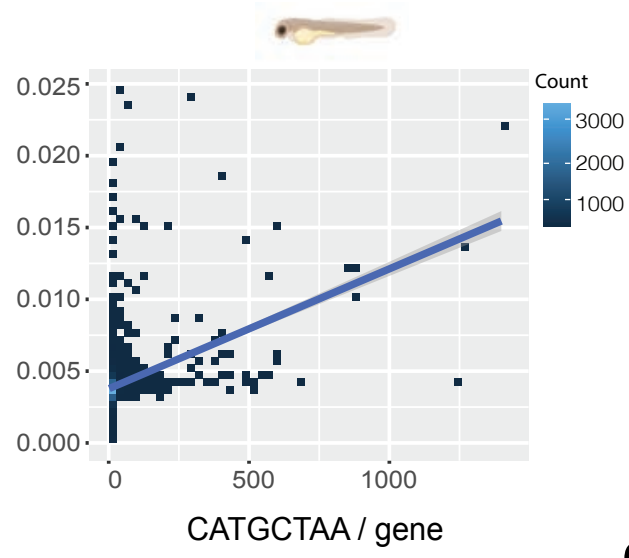

CATGCTAA / gene

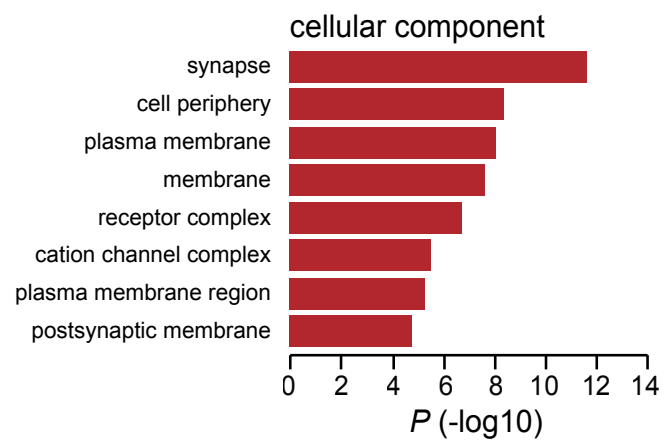

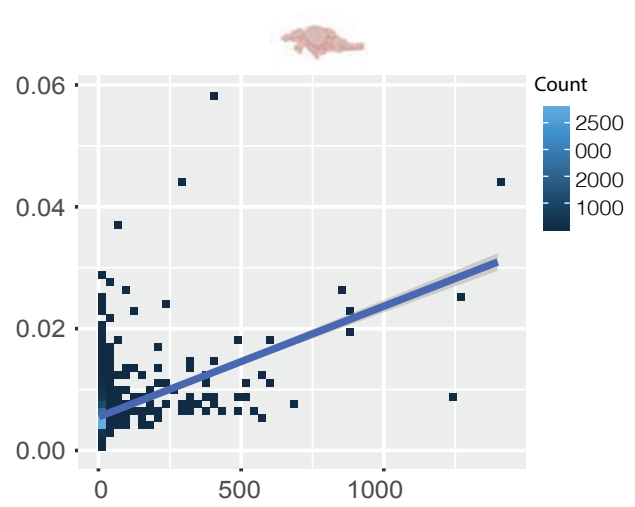

C

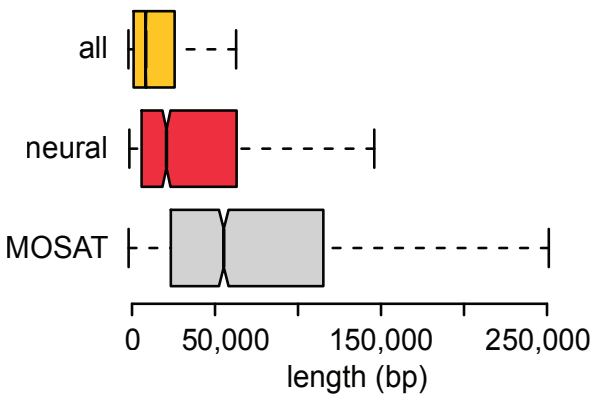

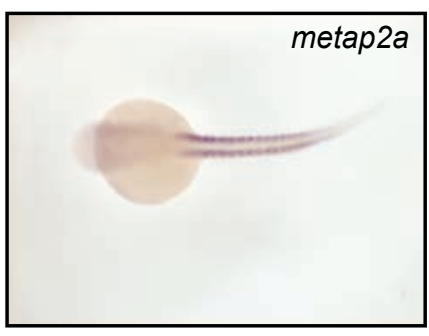
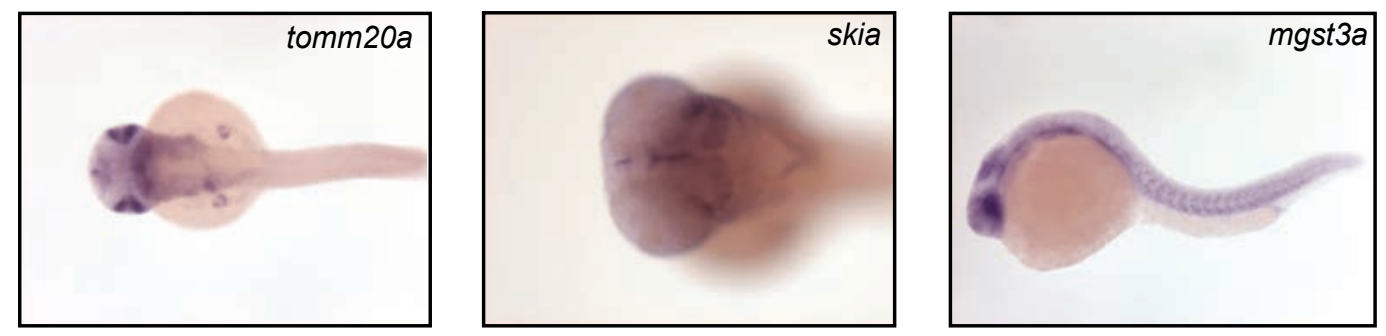

Figure S4 a Scatterplots of average gene methylation $(\mathrm{mCH} / \mathrm{CH})$ plotted againsy the number of MOSAT motifs in the gene. Left to Right: $24 \mathrm{hpf}$ embryo, 48hpf embryo, adult brain. b Gene ontology (GO) analysis of MOSAT motif-containing genes. c Boxplots of average gene lengths of MOSAT motif-containing genes, neural and all genes. d in situ hybridisation of MOSAT motif-containing genes (ZFIN database) in zebrafish embryos showing expression of metap2a in myotomes (fast muscle fibres), expression of tomm20a in retina, tectum, branchial arches and liver, expression of skia in the ventral telencephalon, lens, tectum and branchial arches, and expression of mgst $3 a$ in the eye, tectum and somites. 


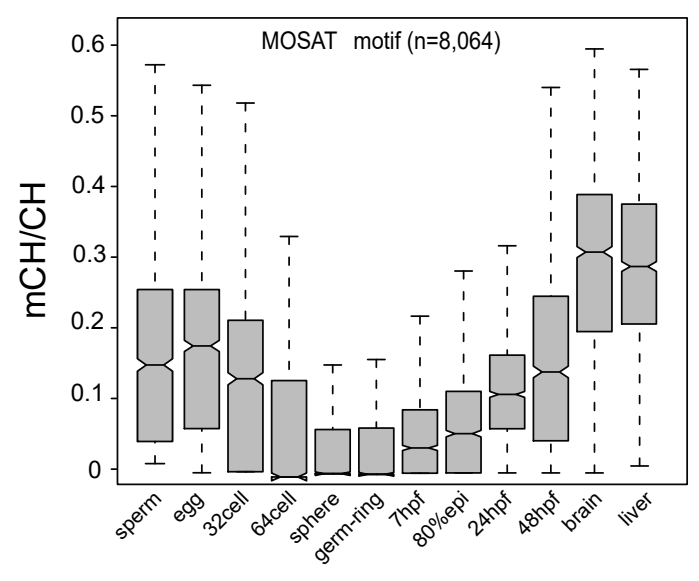

d

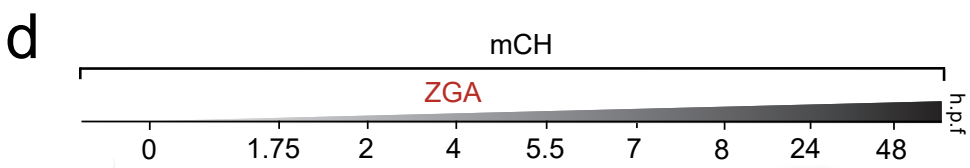
availdo under aCC-BY-NC-ND $4.8,064$ nternationath ligigese.
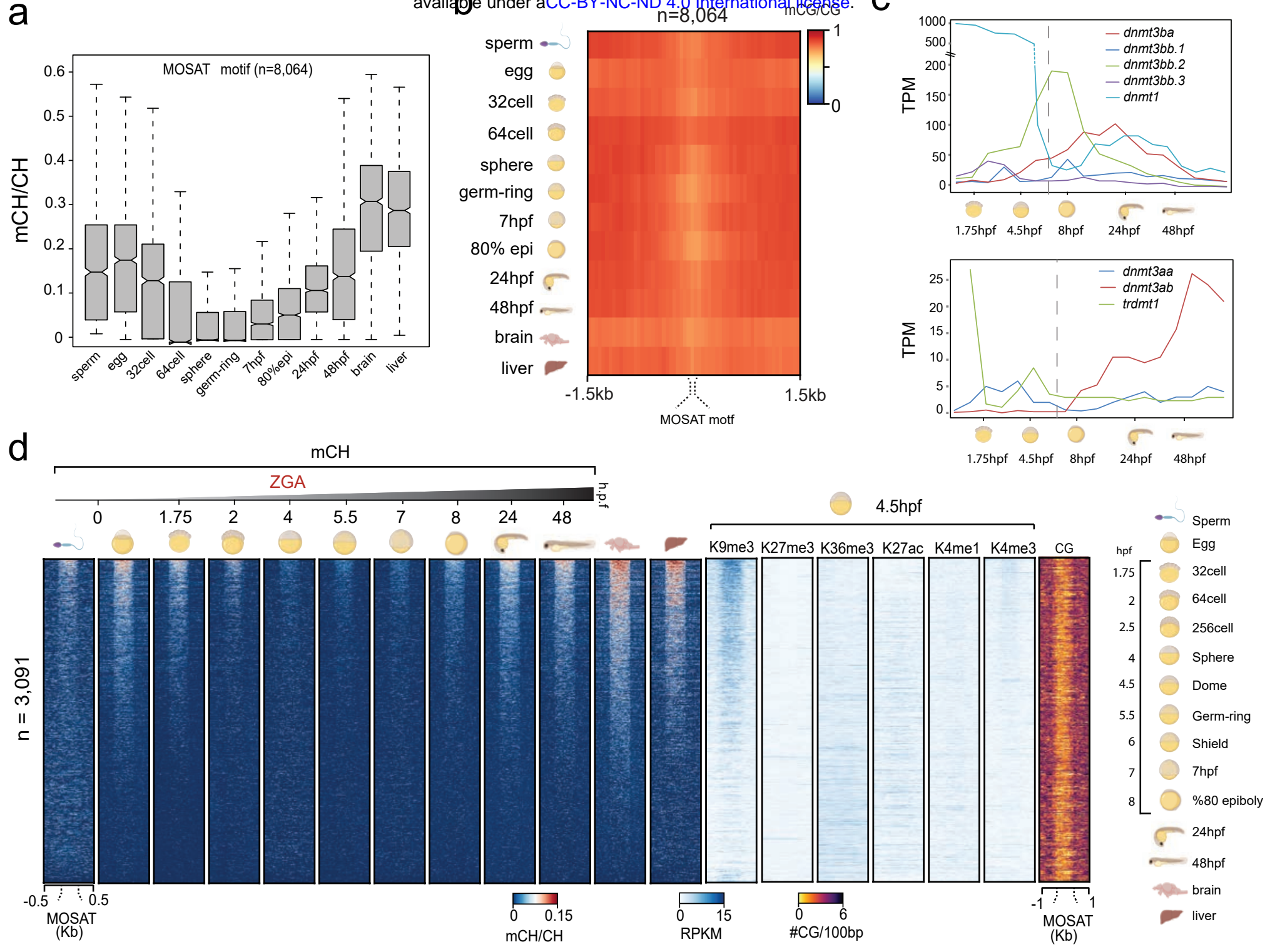

e

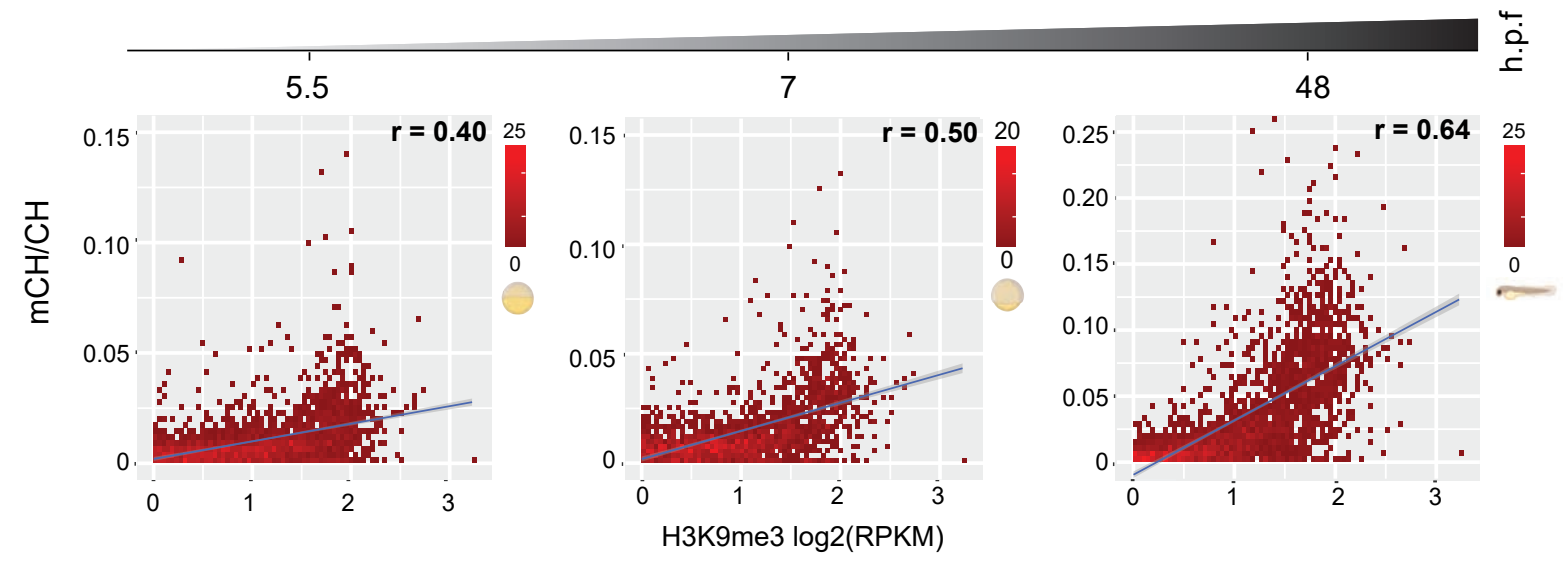

Figure S5. a Boxplots of average mCH levels of MOSAT motifs $(n=8,066)$ in developing zebrafish corrected for bisulfte non-conversion rates determined by unmethylated lambda genome spike in controls. $\mathbf{b}$ mCG profiles of MOSAT motifs $(n=8,064)$ in commonly covered MOSAT_DR elements during zebrafish development. c RNA expression analysis of DNMTs during zebrafish development. Dotted lines indicate the estimated time point of $\mathrm{mCH}$ re-establishment at MOSAT motifs.d Heatmap of $\mathrm{mCH}$ levels and histone (RPKM) levels in MOSAT_DR elements $(n=3,091)$ during zebrafish development. e Scatterplots showing the correlation between $\mathrm{mCH}$ levels at various time points and $\mathrm{H} 3 \mathrm{~K} 9 \mathrm{me} 3$ at shield stage embryos. Left to right: sphere, $7 \mathrm{hpf}, 48 \mathrm{hpf}$. 